Ireneusz Recław, Institute of Mathematics, University Gdansk, Poland.

e-mail: reclaw@math.univ.gda.pl

\title{
ON A CONSTRUCTION OF UNIVERSALLY NULL SETS
}

\begin{abstract}
We describe a new method of constructing universally null sets and universally meager sets using families of Borel sets well-ordered by inclusion.
\end{abstract}

There are several methods of constructing universally null sets. For an overview of some of them and for more references see [3]. In this paper we present another method which looks somehow simpler than others and has interesting consequences.

Let us recall that $X \subset \mathbb{R}^{n}$ is universally null (universally measurable) if $X$ is null (measurable) with respect to each Borel continuous measure on the real line.

We would rather use the following equivalent definitions: $X \subset \mathbb{R}^{n}$ is universally null (universally measurable) if $f[X]$ is of Lebesgue measure zero (is Lebesgue measurable) for each Borel isomorphism $f: \mathbb{R}^{n} \rightarrow \mathbb{R}$.

The dual class for the case of category is the class of perfectly meager sets. $X \subset \mathbb{R}^{n}$ is perfectly meager if $X \cap D$ is meager in $D$ for each perfect set $D \subset \mathbb{R}^{n}$.

E. Grzegorek (see [1]) defined a bit stronger (at least under $\mathrm{CH}$ ) property which was proved to be equivalent to the following one (see [5]). $X \subset \mathbb{R}^{n}$ is universally meager (universally Baire) if $f[X]$ is meager (has Baire Property) for each Borel isomorphism $f: \mathbb{R}^{n} \rightarrow \mathbb{R}$.

The following result strengthens a result of S. Plewik (see [4]).

Theorem 1. Assume that $\mathcal{R} \subset \mathbb{R} \times \mathbb{R}$ is universally measurable (universally Baire) relation. Then any set $X \subset \mathbb{R}$ well ordered by $\mathcal{R}$ is universally null (universally meager).

\footnotetext{
Key Words: universally null, universally meager, coanalitic relation.

Mathematical Reviews subject classification: 03E15,28A05

Received by the editors October 25, 2000

* The author was supported by KBN grant 2P03A 03214
} 
Lemma 1. Suppose $\mathcal{R} \subset \mathbb{R} \times \mathbb{R}$ is Lebesgue measurable (has the Baire property). Then any set $X \subset \mathbb{R}$ well ordered by $\mathcal{R}$ has measure zero (is meager).

Proof. Suppose to the contradictory that $X$ does have positive outer measure. We can assume that for any $y \in X,\{x \in X: x \mathcal{R} y\}$ is a null set. If not, we can consider $\{x \in X: x \mathcal{R} y\}$ where $y$ is the least element of $X$ for which this set does have positive outer measure. Let $A \supset X$ be a Borel set such that $m_{*}(A \backslash X)=0$ and let

$$
W=\left\{(x, y) \in A^{2}: x \mathcal{R} y\right\}
$$

$W$ is a measurable subset of the plane.

Claim. $W$ has planar measure zero.

Proof. Let $V \subset W$ be a Borel subset of $W$ with the same planar measure. The set $\left\{y: m\left(V^{y}\right)>0\right\}$ is Borel, since $V$ is. We will show that it is contained in $A \backslash X$. Let $y \in X$ and $C \supset\{x \in X: x \mathcal{R} y\}$ be a Borel null set. Then $V^{y} \backslash C \subset A \backslash X$ so it is null and $V^{y}$ is null. Thus the set $\left\{y: m\left(V^{y}\right)>0\right\}$ as a Borel subset of $A \backslash X$ is null, so $V$ and $W$ are null on the plane.

But observe that for any $x \in X, W_{x} \supset\{y \in X: x \mathcal{R} y\}$ is of positive outer measure. This contradicts Fubini's Theorem. In the category case we use the Kuratowski-Ulam Theorem.

To prove Theorem 1 just reduce it to the lemma using the Borel isomorphism $f$.

Fact 1. Let $B \subset \mathbb{R} \times \mathbb{R}$ be a Borel set. Then the relation $\mathcal{R}=\left\{(x, y): B_{x} \subset\right.$ $\left.B_{y}\right\}$ is coanalitic so universally measurable and universally Baire.

This gives new method of constructing of universally null sets and universally meager sets.

Theorem 2. Let $\alpha<\omega_{1}$ and $B \subset \mathbb{R} \times \mathbb{R}$ be a universal set for $\Sigma_{\alpha}^{0}(\mathbb{R})$. Assume that $\left\{A_{\beta}: \beta<\kappa\right\} \subset \Sigma_{\alpha}^{0}(\mathbb{R})$ is well ordered by inclusion. Let $x_{\beta} \in \mathbb{R}$ with $B_{x_{\beta}}=A_{\beta}$. Then $\left\{x_{\beta}: \beta<\kappa\right\}$ is universally null and universally meager.

Let us recall a notation $\operatorname{add}(\mathcal{J})=\min \{|\mathcal{F}|: \mathcal{F} \subseteq \mathcal{J} \wedge \bigcup \mathcal{F} \notin \mathcal{J}\}$.

Corollary 1. Let $\mathcal{J}$ be a $\sigma$-ideal containing singletons with a Borel basis and $\operatorname{add}(\mathcal{J})=\kappa$. Then there is a universally null and universally meager set of size $\kappa$. 
Proof. Since for $\kappa=\omega_{1}$ the result is already known we can assume that $\kappa>\omega_{1}$. Observe that $\kappa$ is regular. By transfinite induction we construct the set $\left\{z_{\alpha}: \alpha<\kappa\right\}$ and a family of Borel sets $\left\{A_{\alpha}: \alpha<\kappa\right\}$ in the following way: Let $z_{\alpha}$ be any element of $\mathbb{R} \backslash \bigcup_{\beta<\alpha} A_{\beta}$ and since $\bigcup_{\beta<\alpha} A_{\beta} \cup\left\{z_{\alpha}\right\}$ is in $\mathcal{J}$ let $B$ be any Borel set from the ideal containing $\bigcup_{\beta<\alpha} A_{\beta} \cup\left\{z_{\alpha}\right\}$. By regularity of $\kappa$ we can assume that there is $\alpha<\omega_{1}$ such that $\left\{A_{\alpha}: \alpha<\kappa\right\} \subset \Sigma_{\alpha}^{0}(\mathbb{R})$ Then the family $\left\{A_{\alpha}: \alpha<\kappa\right\}$ satisfies the assumption of Theorem 2 .

In several models of Set Theory it was shown that there is no universally null set or there is no universally meager set of size continuum. For example, it holds in the Cohen real model (universally meager case), the random real model (universally null case) and the iterated perfect set model (both cases) (see [3]). So in these models each $\sigma$-ideal with Borel basis containing singletons has additivity smaller than continuum or equivalently, there is no family of Borel sets well ordered by inclusion of size continuum. In the case of the Cohen real model it was proved by K. Kunen (see [2]).

\section{References}

[1] E. Grzegorek, On sets always of the first category,II, Proceedings of the 13th winter school on abstract analysis (Srnm, 1985), Rend. Circ. Mat. Palermo (2) Suppl. no. 10, (1985), 43-48.

[2] K. Kunen, Inaccessibility properties of cardinals, Doctoral Dissertation, Stanford University.

[3] A.W. Miller, Special subsets of the real line, Handbook of set-theoretical topology, K. Kunen, J. E. Vaughan eds., Elsevier Science Publishers B.V., 1984 .

[4] S. Plewik, Towers are universally measure zero and always of first category, Proc. Amer. Math. Soc., 119(1993), no. 3, 865-868.

[5] P. Zakrzewski, On universally meager sets, preprint. 
